Revue européenne des migrations internationales

vol. $27-n^{\circ} 1 \mid 2011$

La construction de l'altérité dans l'espace noir atlantique : Etats-Unis - France - Caraïbes - Amérique latine

\title{
Éditorial : Contextualiser pour mieux conceptualiser la racialisation
}

Christian Poiret, Odile Hoffmann et Cédric Audebert

\section{(2) OpenEdition \\ Journals}

Édition électronique

URL : https://journals.openedition.org/remi/5283

DOI : $10.4000 /$ remi.5283

ISSN : $1777-5418$

Éditeur

Université de Poitiers

Édition imprimée

Date de publication : 1 juin 2011

Pagination : 7-16

ISBN : 979-10-90426-00-9

ISSN : 0765-0752

\section{Référence électronique}

Christian Poiret, Odile Hoffmann et Cédric Audebert, «Éditorial : Contextualiser pour mieux

conceptualiser la racialisation », Revue européenne des migrations internationales [En ligne], vol. 27 -

$n^{\circ} 1$ | 2011, mis en ligne le 01 septembre 2011, consulté le 14 avril 2022. URL : http://

journals.openedition.org/remi/5283; DOI : https://doi.org/10.4000/remi.5283 


\section{Éditorial}

\section{Contextualiser pour mieux conceptualiser la racialisation}

\section{Christian POIRET ${ }^{1}$, Odile HOFFMANN ${ }^{2}$, Cédric AUDEBERT ${ }^{3}$}

Ce numéro est le fruit d'une réflexion collective ${ }^{4}$. Il vise à restituer un
dialogue interdisciplinaire dans lequel la discussion théorique s'alimente et s'enrichit d'expériences contemporaines de recherche empirique en Amérique latine, en France et aux États-Unis, centrées sur des populations pouvant être catégorisées, selon les contextes, comme « noires », « africaines », « afrodescendantes », " afrocaribéennes », « antillaises », etc.

Cette réflexion s'est développée autour de deux enjeux principaux qui structurent l'ensemble des articles de cette livraison de la REMI. D'une part, il s'agissait de dépasser le « paradoxe racial », c'est-à-dire la contradiction entre l'inexistence avérée des races humaines et l'existence constatée de groupes humains racialisés. Historiciser et (re) localiser les processus d'altérisation et de minorisation à l'œuvre dans divers contextes de l'espace atlantique, ouvre la voie à une dénaturalisation de ces groupes. C'est pourquoi nous avons cherché à appréhender ces processus comme des enjeux contestés et des ressources mobilisables par des groupes, inscrits dans des rapports de pouvoir. D'autre part, parce que les modalités de ces dynamiques d'altérisation et de minorisation varient

1 Maître de conférences HDR en sociologie, Université Paris Diderot, Sorbonne Paris Cité, Unité de Recherche Migrations et Société (URMIS, Universités Paris 7 - Nice, IRD, CNRS) ; poiret@ univ-paris-diderot.fr

2 Géographe, Directrice de Recherche IRD, URMIS-IRD/Université de Paris Diderot, UMR 205 Migrations et société, 159 rue Nationale, 75013, Paris ; odile.hoffmann@ird.fr

3 Chercheur CNRS-Migrinter, Migrinter, MSHS - Bâtiment A5, 5 rue Théodore Lefebvre, 86000 Poitiers ; cedric.audebert@univ-poitiers.fr

4 L'ensemble des articles présentés ici, est issu du colloque Racialisation et ethnicisation : contextes socio-historiques et enjeux contemporains organisé à l'Université Paris-Diderot en novembre 2009 dans le cadre des programmes ANR Afrodesc (ANR-07-SUDS-008) «Afro-descendants et esclavages : domination, identification et héritages dans les Amériques (XVe-XXIe siècles) » (http:// www.ird.fr/afrodesc/) et du programme européen Eurescl (7ème PCRD) « Slave Trade, Slavery, Abolitions and their Legacies in European Histories and Identities » (http://www.eurescl.eu). 
selon les contextes, y compris pour un même groupe, nous voulions réfléchir à la pertinence analytique et à l'utilité opératoire de la distinction entre ethnicisation et racialisation. La confrontation de ce cadre théorique à l'analyse empiriquement fondée des processus en jeu, de leurs contours et de leurs effets, vise donc à éclairer le rapport complexe entre ces deux concepts.

\section{LE PARADOXE RACIAL COMME OBJET DE RECHERCHE}

« Les races humaines n'existent pas », la communauté scientifique s'accorde pour l'affirmer. La diversité biologique et culturelle du genre humain est indéfinie ; elle se décline en arrangements génétiques et en dispositifs culturels dont la complexité est impossible à réduire en quelques " conglomérats 》 ou " groupements " aux frontières figées. Impossible, si ce n'est par un travail de l'esprit qui accorde à tel ou tel trait ou caractéristique, une pertinence plus grande qu'à d'autres, en fonction d'objectifs qui sont en général d'ordre économique, idéologique ou politique.

C'est ce travail d'élaboration qui a été réalisé par les penseurs des Lumières, dès le XVIIIe siècle, au cours d'un vaste procès d'ordonnancement du monde. En cherchant à classifier le vivant et à lui donner un ordre, la pensée racialiste s'est déployée pour distinguer et classer végétaux et animaux. Mais, en s'étendant aux groupes humains, ces principes classificatoires se sont transformés en principes de valeur se voulant explicatifs de l'histoire de l'humanité. Associée à une hiérarchie qui valorise certains plus que d'autres - pour des raisons variant d'un endroit à l'autre, d'une époque à l'autre -, l'idée de « race », au sens moderne du terme, appliquée au genre humain a accouché d'un racisme à prétention scientifique. Dans le cadre plus général d'un « désenchantement du monde », il a pris le relais des justifications théologiques et philosophiques des siècles précédents, pour légitimer par un fondement en nature, la domination et l'exploitation des « inférieurs ». La mise en esclavage n'en est qu'un exemple des plus violents. Distinction, hiérarchisation, domination, exploitation économique et légitimation discursive furent et sont ainsi les piliers du racisme dérivé du racialisme scientifique des XVIIIe et XIXe siècles.

Le XIXe et la première moitié du XXe siècle ont été les théâtres de tragédies humaines menées « au nom de la race » d'une telle ampleur que celle-ci est devenue une notion intolérable et que le racisme a fait l'objet d'une condamnation quasi-universelle. Les idées racialistes ont volontairement et explicitement disparu des argumentaires politiques des pays occidentaux, en particulier en Europe, tandis que des institutions supranationales comme l'UNESCO engageaient des campagnes de décrédibilisation et de condamnation du racisme dès 1945. Pourtant, outre la persistance de génocides et de formes brutales de domination orientés par des principes explicitement racistes tout au long de la deuxième moitié du XXe siècle, la notion de « race » est restée utilisée dans de nombreux pays tels que les États-Unis, le Royaume-Uni ou l'Allemagne, dans un effort de gestion de la diversité des populations (revues et instituts travaillant sur la « race », législation et politiques se référant à des catégories raciales, etc.). Pour sa part, la République française a choisi de supprimer toute référence à la « race » dans les textes officiels, en réaffirmant une idéologie assimilationniste et intégrationniste volontairement aveugle aux origines. 
Ce début de XXIe siècle est marqué par une situation contradictoire. Dans plusieurs contextes nationaux où des catégories raciales ont été instituées à des fins de lutte contre les discriminations, cette démarche fait l'objet de contestations comme, par exemple, la remise en question de la notion de " minorités visibles » au Canada ou des catégories raciales officielles aux États-Unis. Mais simultanément, dans d'autres pays ayant officiellement banni le registre de la « race », plusieurs éléments convergent pour le réintroduire dans les débats. C'est là le phénomène que nous qualifions avec d'autres de racialisation, c'est-à-dire une tendance à interpréter les situations à travers le prisme de la « race » et à agir en conséquence. Face à cette dialectique entre rejet et permanence de la « race ", il faut prendre acte des contradictions à l'œuvre et comprendre pourquoi l'idée de race perdure alors qu'elle est officiellement rejetée (cas de la France) ; comment elle signifie hiérarchie et exclusion alors qu'elle est censée être neutralisée dans un usage administratif (cas des États-Unis) ; ou encore, comment elle apparaît relativisée dans les faits par le métissage alors même qu'elle émerge avec force dans certains contextes de mobilisation sociale (Amérique latine). Bref, il nous faut comprendre comment elle est en permanence resignifiée et intégrée à de nouvelles logiques sociales.

\section{LES CHAMPS DE LA RACIALISATION}

Les champs de la racialisation couvrent un large spectre d'utilisation, consciente ou non-consciente, des registres de la « race » ou de « l'ethnie » dans notre vie quotidienne. Pour illustrer cette prolifération, nous pouvons évoquer, de façon non exhaustive ni hiérarchisée, plusieurs domaines où ces registres trouvent à se déployer quotidiennement. Dans les discours tout d'abord, un besoin de reconnaissance est exprimé par les sujets dominés et exclus de la normativité majoritaire, pour lesquels la revendication de la « différence » peut être un moyen d'accès à plus de justice et de dignité : femmes et hommes qui, du fait de leur phénotype, sont victimes du racisme quotidien et de la stigmatisation ; Indiens las d'être toujours au bas de l'échelle économique et sociale ; « Noirs », " Arabes » ou « Gitans », migrants ou non, systématiquement rabaissés, etc. Sans confondre ni amalgamer des phénomènes divers, on ne peut passer sous silence l'effervescence et le foisonnement actuels d'acteurs, individuels et collectifs, qui demandent une reconnaissance sociale et politique liée à leurs « différences », qu'elles soient fondées sur la couleur, l'origine ou la culture.

La biométrie et la sécurité nationale s'incarnent dans diverses pratiques racialisantes ; avis de recherche pour interpellation de la police française, faisant mention de la caractéristique « R.N. »- race noire - dans un pays qui interdit pourtant l'usage officiel de cette catégorie ; « sans-papiers » potentiels, soupçonnés de séjourner irrégulièrement sur le territoire national, traqués en permanence sur la base de leur apparence - la chasse au faciès -, racialisés selon leur couleur de peau mais aussi leur phénotype, leur hexis corporel ou leur apparence vestimentaire (Goris, Jobard et Levy, 2009). Dans le discours de la sécurité nationale, le danger vient toujours d'alter qu'il faut étiqueter, labelliser, catégoriser, classifier, comme aux plus belles heures du positivisme scientifique du XIXe siècle.

Un autre champ potentiellement racialisé concerne le marché qui, loin d'agir aveuglément, scrute les « niches ethniques » de consommation et développe en consé- 
quence des stratégies de publicité ou de communication destinées à toucher tel public, de telle couleur ; non seulement pour les cosmétiques (shampoing, crèmes, etc.) mais, désormais, aussi pour les vêtements, les loisirs, etc.

La génétique et la médecine évoluent dans le même sens, en développant des traitements sophistiqués de maladies, spécifiques selon la couleur de peau, les origines et/ou l'imputation d'appartenance des malades à des groupes " raciaux ». Elles semblent ainsi renouveler une approche segmentée de la santé/maladie, rappelant l'hygiénisme du XIXe siècle - le malade-pauvre-indigent - et s'appuyant sur « le gouvernement des corps » analysé par Michel Foucault (1997).

Paradoxe, la « race » n'existe pas, mais il est impossible de ne pas la voir opérer partout autour de nous. Il faut donc tout à la fois refuser la réification et la légitimation de « la chose » (la « race ») tout en reconnaissant l'acuité des phénomènes qui sont orientés par la croyance, explicite ou non, en son existence. Bref, il faut « sortir de la race et du racisme » pour mieux les comprendre et en tenir compte pour mieux les déconstruire. Le paradoxe devient alors l'objet même de la recherche.

Autre difficulté, sinon paradoxe, les comportements racialisés opèrent toujours en croisement - en " intersection »- avec d'autres clivages, d'autres hiérarchies : la classe, le genre, la religion, l'âge, l'origine régionale ou nationale, etc. Parties, pour l'essentiel, du mouvement féministe noir-américain, les théories de l'intersectionalité peuvent aider à distinguer les différents processus de domination et à reconnaître leur autonomie relative, tout en prenant en compte leurs déterminations mutuelles. Au lieu de concevoir les effets des différents rapports de pouvoir sur un mode purement cumulatif, il nous faut penser leur articulation et leur interdépendance. Plus qu'une extension sans fin du concept de « racialisation », il s'agit de comprendre comment elle active ou est activée par d'autres modes d'altérisation et de hiérarchisation avec lesquels elle se combine. Ceci suppose de la replacer à chaque fois dans des échelles d'altérité : échelles verticales (la nation, la région, la communauté, le voisinage, l'entreprise) et horizontales (genres, classes, cultures, religions, etc.).

\section{DISTINGUER ETHNICISATION ET RACIALISATION}

C'est dans cette optique que nous avons cherché à distinguer, dans le titre de ce numéro, la racialisation et l'ethnicisation. L'une comme l'autre désignent des modalités de construction de l'altérité dans le cadre de rapports sociaux de pouvoir qui produisent des « Eux » et des « Nous » hiérarchisés, par référence aux origines. Indépendamment des catégories de désignation utilisées, qui peuvent être plus ou moins euphémisées, plus ou moins explicites, les processus d'ethnicisation et de racialisation se distinguent par leurs manières de fabriquer de la « différence ».

L'ethnicisation se réfère à de la culture, au sens anthropologique du terme, acquise et transmise, et elle n'est pas forcément liée à des rapports de domination actuels. Dans sa forme symbolique, elle peut relever d'un mouvement d'auto-identification dans le cadre de jeux de distinction qui n'ont pas de conséquences contraignantes sur la vie des individus, comme dans le cas des Italo-américains ou des Irlando-américains, même 
si elle peut se référer à une mémoire ou à une histoire de la subordination. Lorsqu'elle se combine avec des rapports de pouvoir, elle peut être imputation et servir à désigner des groupes qui sont les cibles de pratiques d'expulsion ou d'assimilation forcée (certains groupes de migrants par exemple) ou d'extermination (guerre civile yougoslave). Mais elle peut être aussi revendiquée, notamment comme un moyen de résister à des processus de racialisation, comme par exemple le refus de la catégorie « Noirs » comme forme d'identification collective par certaines associations d'originaires d'Outre-mer en France métropolitaine, qui lui opposent les identités diverses des territoires d'origine. L'ethnicisation a donc partie liée avec les concepts de nation, de citoyenneté, d'appartenance nationale, toutes choses évidemment contenues également dans la problématique de la racialisation (Balibar et Wallerstein, 1988) mais qui ne lui sont pas forcément consubstantielles comme elles le sont à l'ethnicisation. Ainsi, si l'on adhère à la thèse de " l'école de 1492 », l'idée de race - et donc la source du racisme moderne - émerge de façon concomitante dans la constitution des États nationaux européens et l'expansion coloniale européenne liée aux « grandes découvertes » (Simon, 1970). La première s'est fondée sur une conception exclusive et raciste de l'appartenance aux États-nations, en particulier avec l'édiction de la règle de la pureté de sang après la Reconquista espagnole et l'impossible conversion des supposés Maurisques et Marranes qui en découle. La seconde - l'expansion coloniale s'est construite sur la mise en esclavage et la traite de millions d'êtres humains. Or, si dans le cas de la constitution des minorités européennes, comme les Juifs, la question nationale est immédiatement présente, elle ne l'est pas d'emblée dans le cas de la colonisation qui ne la verra émerger que plusieurs siècles plus tard.

La racialisation se réfère aux pratiques et aux représentations racistes qui, selon les contextes, reposent sur une interprétation des apparences physiques censées traduire des origines communes ; mais aussi sur celle du lignage biologique supposé, indépendamment des caractéristiques phénotypiques exhibées par ceux qui en sont la cible, comme dans le cas du principe de la goutte de sang aux États-Unis ou des imputations de judéité en Europe. Dans un cas comme dans l'autre, la racialisation est fondée en nature, elle relève de l'hérédité et non de l'héritage, et elle s'inscrit toujours dans des rapports de domination/subordination contraignant la vie quotidienne des individus. Même si le processus de racialisation peut comprendre un mouvement de retournement du stigmate qui amène les dominés à revendiquer et à retravailler la catégorie qui est au cœur de leur domination, d'un point de vue historique, elle commence toujours par une imputation catégorielle imposée par le groupe dominant. Ce dernier pouvant lui-même, selon les contextes historiques et sociopolitiques, sortir ou non de son indétermination et se revendiquer ou pas comme groupe racisé. Dans ce cadre, l'utilité d'établir un distinguo entre racisation et racialisation ou entre ethnisation et ethnicisation - c'est-à-dire entre face matérielle et face mentale d'un même rapport social - est discutée dans différents articles de ce numéro.

La racialisation ne suppose pas forcément l'usage de catégories se référant explicitement à des schèmes de pensée raciologiques (noirs, blancs, brachycéphales, quarterons, mulâtres, caucasiens, etc.) et d'autres catégories sociales - les catégories ethniques (Arabes, Chinois, etc.) et religieuses (Juifs, Musulmans, etc.) en particulier - peuvent être investies du sens de la race. Parce qu'elle se réfère à de l'inné et qu'elle construit une altérité insurmontable, tendanciellement, la racialisation ne s'accompagne pas d'injonction à l'assimilation mais plutôt de pratiques de ségrégations et de contention. 
Dans le champ académique, les débats restent ouverts quant à la nature de la distinction entre « race » et ethnicité, notamment en raison de variations de traditions politiques et scientifiques nationales. Certains conçoivent la racialisation comme une forme exacerbée de l'ethnicisation tandis que d'autres considèrent qu'il s'agit de deux dynamiques différentes par essence. Pour notre part, nous avons conceptualisé l'ethnicisation et la racialisation comme deux processus qui expriment et relèvent d'un même type générique de rapports sociaux - généralement désigné en France comme rapports interethniques et comme Race Relations en Grande-Bretagne. Ces rapports sociaux jouent un rôle structurant dans toutes les sociétés contemporaines, bien qu'ils puissent prendre des formes distinctes selon les contextes (ethnocentrisme, xénophobie, ethnisme, racisme, nationalisme, etc.). D'ailleurs il ne s'agit pas de phénomènes totalement étanches, puisque force est de constater qu'un même groupe ou un même individu peut être tour à tour ethnicisé ou racisé selon les lieux, les périodes ou les contextes d'interaction. Notre propos vise donc à préciser la nature de ces deux phénomènes afin de rechercher l'éventuel intérêt heuristique de procéder à une telle distinction.

\section{ETHNICISATION, RACIALISATION ET LOGIQUES D'ACTION}

Les textes ici réunis interrogent donc l'utilité de la distinction entre ethnicisation et racialisation. Ils le font en cherchant à vérifier l'hypothèse selon laquelle le développement de l'un et/ou l'autre de ces processus correspond à des logiques d'action adaptées au contexte dans lequel ils se développent. L'option pour l'un ou l'autre schéma (de pensée et d'action) est toujours contextuelle, stratégiquement élaborée et négociée. Elle répond et traduit des rapports de force et de pouvoir à un moment donné. En particulier, le passage de la prédominance d'une forme d'altérisation à une autre semble lié aux transformations ou aux volontés de transformation de la distance sociale entre les groupes en présence.

Dans le cas français, on peut penser que les tentatives de subsumer les catégories Africains et Antillais sous la catégorie « Noirs » - qui sont loin de faire l'unanimité - s'inscrivent dans le contexte d'une homogénéisation des descendants de migrants et d'un début de fusion dans la population métropolitaine. Ces évolutions rendent moins pertinentes les distinctions ethniques utilisées jusqu'alors, tant du côté majoritaire pour construire de la distance et pour orienter des politiques de gestion spécifiques, que du côté minoritaire pour aménager ou contester leur condition. La société française semble donc être le théâtre d'une racialisation croissante des populations pouvant être catégorisées comme « noires ", dans le contexte d'une intégration nationale où, dans certaines circonstances, les distinctions ethniques en vigueur jusqu'alors sont rendues moins pertinentes. Ces dernières le sont en revanche plus que jamais parmi les « Noirs » aux États-Unis, pays où les immigrations caribéenne et africaine connaissent une ampleur et un renouvellement sans précédent, tandis que les catégories raciales instituées font l'objet de mouvements de contestation.

Dans ces deux contextes nationaux nettement distincts, on peut constater l'existence locale d'associations ethniques territorialisées entrant en concurrence ou en complémentarité avec des structures se référant à une identité raciale au niveau national - ou tout au moins avec des tentatives pour les faire exister. 
Dans le cas des États-Unis, en déplaçant la focale de la catégorie raciale en vigueur au niveau national vers les communautés ethniques locales, les stratégies mises en œuvre par les migrants redéfinissent le sens d'une identité caribéenne et questionnent la catégorisation ethno-raciale instituée. La comparaison est intéressante avec le contexte français. Elle met en lumière des dynamiques à contre-courant de celles observées aux États-Unis, avec des tentatives d'affirmation à une échelle nationale de formes d'organisation fondées sur une identité racisée qui entrent en concurrence avec des modes d'identification ethnique préexistants, s'appuyant en grande partie sur un tissu d'organisations locales.

Le rapprochement avec les contextes mexicain, centraméricain et colombien est également instructif, en particulier lorsqu'on le remet en perspective historique. Sur le temps long, avec un certain décalage, la genèse des catégorisations et leurs incidences révèlent des similitudes entre les divers contextes américains, liées à l'histoire de la colonisation sur ce continent. On peut, par exemple, constater des tentatives pour ethniciser des populations qui étaient jusqu'alors racisées. Pour autant, trois éléments contextuels distinguent fondamentalement les situations états-unienne et latino-américaine - ellesmêmes très diverses - et agissent différemment sur les processus catégoriels. Les ÉtatsUnis restent le berceau d'une lutte pour les droits civiques des Noirs et de schèmes de distinction officialisés qui ont profondément marqué l'identité et les bases de la cohésion sociale états-unienne. De plus, l'importance d'une immigration continue et diversifiée comme modalité de construction permanente des États-Unis a produit un contexte pluriculturel singulier, complexifiant les identités collectives et la relation qu'elles sous-tendent entre catégories « raciales » et ethniques. Enfin, la place majeure conférée au métissage dans la construction des mythes nationaux de plusieurs pays latino-américains - non sans ambiguïté - tranche avec son absence dans les discours, les idéologies et les pratiques institutionnelles ayant fondé la société états-unienne. Cette mise à l'écart officielle du métissage a profondément et durablement pesé sur les processus de racialisation et d'ethnicisation des populations noires de ce pays.

Le rapprochement entre les contextes nationaux fort distincts qui sont analysés dans ce numéro tend à montrer le caractère opératoire et la fonction stratégique de la distinction entre dynamiques d'ethnicisation et de racialisation, de même que la complémentarité de ces processus dans les luttes ayant pour enjeu l'accès des groupes concernés aux ressources, au pouvoir et à la représentation politique. C'est d'ailleurs le recours à cette problématique en termes de processus de construction de l'altérité, selon des modalités et des fonctionnalités variables, qui autorise de possibles comparaisons entre des contextes nationaux et historiques pourtant extrêmement contrastés.

\section{L'ESPACE ATLANTIQUE COMME LABORATOIRE D'ÉTUDE}

L'originalité de ce numéro réside dans la mise en perspective et la confrontation de paradigmes et d'approches élaborés dans des contextes politiques, sociaux et intellectuels différents. L'espace atlantique est en effet un laboratoire exceptionnel pour comprendre les processus de racialisation et d'ethnicisation de populations issues d'Europe, d'Afrique et des Amériques. Les sociétés locales, inscrites dans des circulations multiples comme 
dans des espaces et des histoires particuliers, ont généré des schémas spécifiques de hiérarchisation et d'exclusion/inclusion. Le rapprochement de divers cadres nationaux interroge ainsi les mécanismes sociaux, politiques et institutionnels de production de l'altérité et les différents types de rapport au territoire qu'ils produisent. Car il s'agit bien de mécanismes concrets de « fabrication nationale », forgés dans le local-quotidien comme dans le globalidéologique (même si le quotidien ne saurait s'opposer à l'idéologique).

En outre, les désignations racisantes et ethnicisantes sont apparues pour qualifier l'Autre soumis, subalterne. Elles reflètent une relation - fût-elle mémorielle - au dominant, au majoritaire, au colonial. Mais, selon les époques et les lieux, elles ont évolué jusqu'à produire aujourd'hui une terminologie foisonnante. Ces désignations catégorielles ont une charge performative très efficace, elles façonnent autant qu'elles rendent compte de nos sociétés. Les catégories employées doivent donc être rapportées au point de vue de qui les subit ou de qui les utilise comme armes. D'où notre volonté de faire dialoguer des cadres théoriques et des cas concrets, ethnographiés ou documentés.

En France, la mobilisation de collectifs comme le CRAN (Conseil Représentatif des Associations Noires) ou les Indigènes de la République, le débat sur les statistiques ethniques, les revendications pour plus de présence sur les scènes publiques (politiques, médias, éducation, culture) ne peuvent être ignorés ni réduits à des préoccupations marginales. En Amérique latine, la situation n'est pas plus simple et elle s'est profondément transformée en moins de vingt ans, au Nord comme au Sud. Là où leur présence contemporaine était méconnue - car pour sa part l'histoire ne les a jamais tout à fait ignorées -, les populations « noires » ont désormais une place et, parfois, un rôle politique majeur. À l'inverse, là où elles participaient activement aux débats, il y a à peine dix ans, elles ont aujourd'hui perdu beaucoup de leurs capacités de négociation et parfois même jusqu'à certains de leurs acquis si chèrement obtenus - comme la maîtrise des territoires collectifs des communautés afrodescendantes en Colombie. Quant au bassin caribéen, on y retrouve à la fois des constructions identitaires héritées de l'esclavage et des modalités liées aux apports migratoires historiques (apports africains, asiatiques, européens, etc.), toutes deux articulées aux expériences migratoires contemporaines vers les métropoles comme le montrent les expériences des Antillais aux États-Unis ou en France.

\section{RÉACTUALISATION DE PROBLÉMATIQUES ANCIENNES ET ÉMERGENCE DE NOUVEAUX QUESTIONNEMENTS}

Cette livraison de la REMI est organisée autour de trois thèmes. Le premier concerne les expressions publiques de l'altérité et leurs conceptualisations. Les enjeux de l'ethnicisation et de la racialisation des populations afrodescendantes, ainsi que les va-et-vient et complémentarités entre les deux processus, sont analysés dans le contexte mexicain par Odile Hoffmann et dans celui des migrations caribéennes aux États-Unis par Cédric Audebert. Carlos Agudelo change d'échelle d'observation en s'intéressant à l'articulation entre transnationalité territoriale, construction identitaire et action politique des Garifunas entre Amérique centrale et Amérique du Nord. 
Le deuxième thème analyse les politiques publiques mises en œuvre, cherchant à en tirer non pas un bilan, mais bien des tendances - éventuellement contradictoires - dans le rapport du politique aux affirmations identitaires. Les cadres politiques de la reconnaissance des populations afrodescendantes et du multiculturalisme sont étudiés dans l'enseignement supérieur colombien par Oscar Quintero, tandis que Gloria Lara analyse la construction politique du « Noir » au Mexique.

Dans une troisième partie, les dynamiques sociales et culturelles des identifications sont abordées selon différentes approches. Christian Poiret interroge la dimension subjective des processus de racisation chez de jeunes femmes «noires » en France métropolitaine. María Elisa Velázquez remet en perspective historique la relation entre l'idéologie du métissage et la place des Afrodescendants dans la construction nationale mexicaine. Elisabeth Cunin se focalise sur le contexte yucatèque en interrogeant la figure des negritos et mestizos dans la première moitié du XXe siècle.

De l'ensemble de ces textes ressortent cinq constats dans les recherches relatives aux processus d'altérisation et de minorisation. Tout d'abord, la nécessité d'historiciser les processus à l'œuvre, à travers la genèse des modes de catégorisation et leurs incidences passage de la distinction à la hiérarchie, puis à l'exploitation économique pour aboutir à la légitimation discursive de la domination - avec comme logique de fond leur inscription dans des rapports de force politiques et économiques.

Ensuite, la compréhension de ces dynamiques historiques de représentation de l'altérité doit être affinée par une analyse en termes d'acteurs : qui produit ces catégories ? À quelles fins ? Selon quelles logiques ? Pour produire quelle forme d'altérité ? Ceci amène logiquement à interroger les champs de déclinaison de la catégorisation produite : les champs politique et académique, mais aussi juridique, culturel ou socio-économique.

Cette double approche soulève la question du décalage entre les discours officiels (en particulier ceux qui nient l'apport des populations afrodescendantes à la construction nationale, comme au Mexique) et les pratiques, le vécu quotidien et les interactions entre les différentes populations catégorisées. Les idéologies et processus institutionnels d'inclusion cohabitent avec des facteurs d'exclusion et de discrimination historiques intériorisés par les sociétés nationales. Ce décalage est propice au développement de contradictions et de tensions dont les effets sur les dynamiques identificatoires des groupes afrodescendants ont été remarquables. Le retournement du stigmate comme mode de gestion de ces contradictions le montre dans différents contextes.

Pour sa part, la spatialisation du regard confère une dimension supplémentaire à l'approche en sciences sociales, à travers l'articulation de trois échelles d'analyse dans la genèse de ces processus identificatoires. La circulation transnationale des catégories et symboles produit des identités inscrites dans des espaces et des histoires plus larges que celles des entités nationales ; mais la circulation est entendue aussi dans certains cas comme la transposition de catégories associées à la situation d'une population à celles d'une autre, à l'instar de la projection de l'appréhension des populations indiennes sur celle des Afrodescendants, dans certains contextes latino-américains. Les agences internationales jouent un rôle accru dans la circulation de ces catégories. Elles ne peuvent 
cependant être étudiées indépendamment de la fonction des processus de racialisation et d'ethnicisation dans la construction des mythologies nationales et leurs avatars régionaux et locaux. L'instrumentalisation du territoire local (quartier, ville) à des fins d'inscription spatiale de l'altérité montre la réinvention à l'échelon local de lignes de clivage ethniques ou raciales devenues moins évidentes à l'échelle nationale.

Enfin, chacun des textes est animé par la problématique sous-jacente des limites ou contours des catégories analysées, et donc celle, indissociable, du métissage. La question de la réalité plus ou moins reconnue du métissage, de son instrumentalisation à des fins politiques ou économiques, et de ses ambiguïtés, est ici centrale. Le métissage, processus biologique et social, est aussi - et surtout - une construction politique qui, dans certains contextes particuliers - celui des indépendances latino-américaines par exemple a servi de base à des mythologies nationales ayant mis à l'écart certaines populations minorisées, en particulier indiennes ou afrodescendantes. Comme hier aux États-Unis, le débat actuel sur les statistiques ethniques en France ou en Colombie réactualise cette question en interrogeant la place du métissage dans les catégorisations politiques validées par les institutions.

\section{Références bibliographiques}

BALIBAR Étienne et WALLERSTEIN Immanuel (1988) Race, Nation, Classe. Les Identités Ambiguës, Paris, La Découverte, 309 p.

FOUCAULT Michel (1997) "Il faut défendre la société ». Cours au Collège de France (19751976), Paris, Seuil/Gallimard, 283 p.

GORIS Indira, JOBARD Fabien et LEVY René (2009) Police et minorités visibles : les contrôles d'identité à Parisi, New York, Open Society Institue, 53 p.

SIMON Pierre-Jean (1970) Ethnisme et racisme ou l'École de 1492, Cahiers Internationaux de Sociologie, vol. XLVIII, 1970, Paris, PUF, pp. 119-152. 